\title{
Evaluation of geometrical characteristics and capacity of vertical cylindrical tanks by least square method
}

Key Words: Tank, geometrical characteristics, geodetic measurement, least square method, shape model

\section{L'evaluation des caracteristiques geometriques et capacite de reservoirs cylindriques verticaux par la methode des moindres carres}

Mots clés: réservoir, caractéristique géométrique, méthode des moindres carrés, modèle de la forme

\author{
Sergii Pronenko and Olexandr Samoylenko \\ SE “Ukrmetrteststandard”, 4, Metrologischna str., Ukraine
}

\begin{abstract}
It has been proposed strict, from least square method (LSM), solution of evaluation geometrical parameters of vertical cylindrical tank (cylindrical part mean radius, tank tilt, axial tilt directional angle, radial deviations of tank wall from approximate cylinder) by point coordinates at the tank wall that are calculated by the measurement results of horizontal and vertical angles and distances by electronic total stations and scanners. For strict record of measurement uncertainty while approximation by LSM, it has been proposed to use their correlation matrix. It has been proposed simple model of evaluation resulting relative uncertainty of tank and course capacity, that include A-type uncertainty, evaluated as standard radial deviation and B-type uncertainty, evaluated on the base of metrological researches of the instruments that are used during measurements.
\end{abstract}

Theoretically the tank wall surface must have cylindrical shape and the axes of this cylinder must be vertical, but in practice it is impossible. As soon as the point coordinates on the real tank wall surface are obtained with the geodetic instrument one could estimate mid radius of cylinder and it axis tilt by processing cylindrical surface approximation. Evaluation of statistic characteristic of tank wall deformation and roughness is fulfilled by radial deviation. Radial deviation is the shortest distance between real and approximation cylindrical surfaces. The other word it is the distance to a real surface toward approximated surface (in this point). The name of this statistic characteristic is standard radial deviation. This statistic characteristic would include uncertainty of geodetic measurements which influence at geometrical parameters uncertainty and therefore at capacities must be estimated. Accuracy of evaluation of standard radial deviation would depend on sample size (points quantity at the surface which coordinates are determined) and statistic characteristics of geodetic measurements. With the use of standard radial deviation evaluation, A-type uncertainty of geometrical parameters and tank capacity is estimated.

So the main goal is to develop strict method (in the sense of least square method) of evaluation geometrical parameters and capacity of vertical cylindrical tanks and their uncertainty according to the results of geodetic measurements.

It has been proposed strict, for the sense of least square method (LSM), solution of evaluation the geometrical parameters of vertical cylindrical tank (cylindrical part mean radius, tank tilt, axial tilt directional angle, radial deviations of tank wall from approximate cylinder) by point coordinates at the tank wall that are calculated by the measurement results of horizontal and vertical angles and distances by electronic total stations and scanners. For strict record of measurement uncertainty while approximation by LSM, it has been proposed to use their correlation matrix. It has been proposed simple model of evaluation resulting relative uncertainty of tank and course capacity, that include A-type uncertainty, evaluated as standard radial deviation and B-type uncertainty, evaluated on the base of metrological researches of the instruments that are used during measurements.

While development mathematically strict generalized method of measurement results processing one can point out such main issues:

1) evaluation of tank surfaces geometrical parameters by approximation of the measured spatial coordinates by least square method (LSM); 
2) evaluation of geometrical parameters uncertainty of tank surfaces by LSM;

3) calculation of tank graduation characteristics (interval capacities) with the use of obtained geometrical parameters and real tank shape toward approximating surface;

4) strict evaluation by LSM of the tank interval capacities uncertainty based on geometrical parameters uncertainty of object surface evaluated by LSM.

Measurement model binding points coordinates on the surface and its geometrical parameters:

$$
\varphi_{i}\left(x_{i}, y_{i}, z_{i}, \tau_{1} \ldots \tau_{k}\right)=0
$$

where $\boldsymbol{x}_{\boldsymbol{i}}, \boldsymbol{y}_{\boldsymbol{i}}, \boldsymbol{z}_{\boldsymbol{i}}$ are horizontal coordinates and absolute height of points on the object surface (where $\boldsymbol{i}=1 \ldots n$ ), which are measured by geodetic instruments;

$\tau_{1} \ldots \tau_{k}$ are defined geometrical parameters of the object surface;

$\boldsymbol{k}$ is quantity of determined geometrical parameters;

$\boldsymbol{n}$ is quantity of points at the surface which coordinates are determined.

Absolute height is vertical distance from horizontal flatness which passes through dip-point to point with number $\boldsymbol{i}$.

Due to surface roughness and coordinate measurement uncertainties equations (1) are not fulfilled. That is why for evaluation of determined geometrical parameters it is necessary to make equations of corrections, which we obtain by partial derivation of measurement model (1) by measured coordinates and defined parameters:

$$
\frac{\partial \varphi_{i}}{\partial x_{i}} v_{x_{i}}+\frac{\partial \varphi_{i}}{\partial_{i}} v_{y_{i}}+\frac{\partial \varphi_{i}}{\partial z_{i}} v_{z_{i}}=\frac{\partial \varphi_{i}}{\partial \tau_{l}} \delta \tau_{i}+\ldots+\frac{\partial \varphi_{i}}{\partial \tau_{j}} \delta \tau+\ldots+\frac{\partial \varphi_{i}}{\partial \tau_{k}} \delta \tau_{k}+l_{i}(2)
$$

where $\boldsymbol{v}_{\boldsymbol{x}_{\boldsymbol{i}}}, \boldsymbol{v}_{\boldsymbol{y}_{\boldsymbol{i}}}, \boldsymbol{v}_{z_{\boldsymbol{i}}}$ are corrections to measured coordinates of point with number $\boldsymbol{i}$ on the surface;

$$
\delta \tau_{1}, \delta \tau_{j}, \delta \tau_{k} \text { are corrections to approximate }
$$
values of the determined parameters $\tau_{1}^{0} \ldots \tau_{k}^{0}$;

$$
l_{i}=\varphi_{i}\left(x_{i}, y_{i}, z_{i}, \tau_{1}^{0} \ldots \tau_{k}^{0}\right) \text { is constant term of }
$$
equation of corrections.

In matrix parametric equation of corrections system (2) has the view:

$$
\begin{aligned}
& \boldsymbol{A} \cdot \boldsymbol{V}=\boldsymbol{B} \cdot \delta \tau+\boldsymbol{l} \\
& \vartheta=\boldsymbol{B} \cdot \delta \tau+\boldsymbol{l},
\end{aligned}
$$

where $\boldsymbol{A}$ is matrix of partial derivatives from measurement model (1) by points measured coordinates; coordinates;

$\boldsymbol{V}$ is correction matrix to points measured

$\vartheta$ is diagonal matrix of radial deviations of the tank real surface from approximating;
$\boldsymbol{B}$ is partial derivatives matrix from measurement model (1) by determined geometrical parameters;

$\delta \tau$ is correction vector to approximate values of determined parameters;

$\boldsymbol{l}$ is constant terms vector of correction equation.

Taking into account that parametric equations of corrections (2) are much more then determined geometrical parameters, one build standard equation system, that in matrix, taking in account that $\boldsymbol{B}^{\boldsymbol{T}} \cdot \boldsymbol{\vartheta}=\boldsymbol{0}$, has the view:

$$
\begin{gathered}
B^{T} \cdot B \cdot \delta \tau+B^{T} l=0 \text { or } \\
N \cdot \delta \tau+L=0 .
\end{gathered}
$$

Corrections to approximate values of the determined geometrical parameters in matrix are obtained by solving system of linear equations (5) by formulas:

$$
\delta \tau=-N^{-1} \cdot L=-Q \cdot L .
$$

where $\boldsymbol{N}^{-1}=\boldsymbol{Q}$ is matrix inversed to standard equation matrix.

Standard radial deviation $\sigma_{\vartheta}$ of the tank real surface from approximate and correlation (covariation) matrix of determined tank surface geometrical parameters are calculated by formulas:

$$
\begin{gathered}
\sigma_{\vartheta}=\sqrt{\frac{\sum_{i=1}^{n} \vartheta_{i}^{2}}{n-k}} ; \\
K_{\delta \tau}=\sigma_{\vartheta}^{2} \cdot Q .
\end{gathered}
$$
LSM:

Received radial deviations fit to main principals of

$$
\sum_{i=1}^{n} \vartheta_{i}=0 ; \quad \sum_{i=1}^{n} \vartheta_{i}^{2}=\min .
$$

To tank geometrical parameters one can refer:

- parameters of its spatial attitude - coordinates of tank center or point on its axis (for calculation of tank capacity are not used but obligatory included into (1) and they are necessary while calculations (2) - (5));

- parameters of its spatial orientation - angles between tank axis and coordinate axis or planes, for example, angle and direction (azimuth) axis tilt of vertical cylindrical tank;

- parameters of its dimensions and shape, for example, radius of spherical or cylindrical tank, minor semiaxis of elliptical tank bottom or height of conic tank bottom.

Geometrical parameters of orientation, dimensions and shape enter into formulas for calculation of total and interval capacities of the tank:

$$
V_{z_{f}}=\psi_{V}\left(\tau_{1} \ldots \tau_{k}, z_{f}\right)
$$

where $\boldsymbol{z}_{\boldsymbol{f}}$ is absolute height of liquid in the tank with current number $\boldsymbol{f}$, for which interval capacity is calculated. 
Evaluation of standard deviation (A-type standard uncertainty) unimproved by the tank interval capacity correction to height $\boldsymbol{z}_{f}$ is calculated by formula:

$$
\begin{gathered}
\boldsymbol{u}_{A}^{2}\left(V_{z_{f}}\right)=\sigma_{V_{z_{f}}}^{2}=\boldsymbol{F}_{V_{z_{f}}} \cdot \boldsymbol{K}_{\delta \tau} \cdot \boldsymbol{F}_{V_{z_{f}}}^{T}=\sigma_{g}^{2} \cdot \boldsymbol{F}_{V_{v_{f}}} \cdot \boldsymbol{Q} \cdot \boldsymbol{F}_{V_{z_{f}}}^{T},(9) \\
\text { where } \boldsymbol{F}_{V_{z_{f}}}=\left|\frac{\partial \psi_{V}}{\partial \tau_{1}} \ldots \frac{\partial \psi_{V}}{\partial \tau_{j}} \ldots \frac{\partial \psi_{V}}{\partial \tau_{\boldsymbol{k}}}\right| \text { is partial }
\end{gathered}
$$

derivatives vector from function (8) by the tank geometrical parameters, that take part in calculation of its capacity or capacity of its part.

In accordance with the first formula from (7) correction to the tank total capacity for radial deviation (terrain correction) of the tank surface toward approximating surface must be equal to zero. However, only such terrain corrections into tank interval capacities allow to exceed in accuracy the volumetric method of measurement for the tanks with total capacity up to $50 \mathrm{~m} 3$.

Taking into account a great number of points, which coordinates are determined by method of laser scanning on tank surface, it is proposed to calculate terrain corrections $\Delta \boldsymbol{V}_{z_{f}}$ by formulas:

$$
\bar{\vartheta}_{z_{f}}=\frac{\sum_{i=1}^{n_{z_{f}}} \vartheta_{i}}{n_{z_{f}}} ; \quad \Delta V_{z_{f}}=S_{z_{f}} \cdot \bar{\vartheta}_{z_{f}},
$$

where $\boldsymbol{S}_{z_{f}}=\psi_{S}\left(\tau_{1} \ldots \tau_{k}, \boldsymbol{z}_{f}\right)$ is the tank surface area to height ${ }^{\boldsymbol{z}_{f}}$;

$$
\begin{aligned}
& \overline{\boldsymbol{\vartheta}}_{z_{f}} \text { is the tank mid radial deviation to height }{ } \boldsymbol{z}_{f} \\
& \boldsymbol{n}_{z_{f}} \text { is a number of points on the tank surface to }
\end{aligned}
$$
height $\boldsymbol{z}_{f}$, which coordinates were determined.

Evaluation of standard deviation (A-type standard uncertainty) of the tank surface area to height $\boldsymbol{z}_{f}$ is fulfilled by formula:

$$
\begin{gathered}
\boldsymbol{u}_{A}^{2}\left(\boldsymbol{S}_{z_{f}}\right)=\sigma_{S_{z f}}^{2}=\boldsymbol{F}_{S_{z f}} \cdot \boldsymbol{K}_{\delta \tau} \cdot \boldsymbol{F}_{S_{z f}}^{T}=\sigma_{\vartheta}^{2} \cdot \boldsymbol{F}_{S_{z f}} \cdot \boldsymbol{Q} \cdot \boldsymbol{F}_{S_{z_{f}}}^{T},(11) \\
\text { where } \boldsymbol{F}_{S_{z_{f}}}=\left|\frac{\partial \psi_{S}}{\partial \tau_{1}} \ldots \frac{\partial \psi_{S}}{\partial \tau_{j}} \ldots \frac{\partial \psi_{S}}{\partial \tau_{\boldsymbol{k}}}\right| \text { is partial }
\end{gathered}
$$

derivatives vector from the tank surface area to height $\boldsymbol{z}_{f}$ by tank geometrical parameters that take part in it calculation.

Standard deviation of mid radius deviation of the tank wall to height $\boldsymbol{z}_{f}$, which is comprised in formulas (10), is calculated by formula:

$$
\sigma_{\bar{\vartheta}_{z_{f}}}=\sqrt{=\frac{\sum_{i=1}^{n_{z_{f}}}\left(\vartheta_{i}-\bar{\vartheta}_{z_{f}}\right)^{2}}{n_{z_{f}}\left(n_{z_{f}}-1\right)}}
$$

Standard deviation (A-type uncertainty) of terrain correction $\Delta \boldsymbol{V}_{z_{f}}$ is calculated by formula:

$$
u_{A}\left(\Delta V_{z_{f}}\right)=\sigma_{\Delta V_{z f}}=\sqrt{\bar{\vartheta}_{z_{f}}^{2} \cdot \sigma_{S_{z_{f}}}^{2}+S_{z_{f}}^{2} \cdot \sigma_{\bar{\vartheta}_{z_{f}}}^{2}}
$$

Adjusted terrain correction of the tank interval capacity to height $\boldsymbol{z}_{f}$ and it uncertainty are calculated by formulas:

$$
\begin{gathered}
\bar{V}_{z_{f}}=V_{z_{f}}+\Delta V_{z_{f}} \\
\boldsymbol{u}_{A}\left(\bar{V}_{z_{f}}\right)=\sqrt{\boldsymbol{u}_{A}^{2}\left(V_{z_{f}}\right)+u_{A}^{2}\left(\Delta V_{z_{f}}\right)} .
\end{gathered}
$$

For vertical tank the measurement model (1) has the view:

$$
R=\sqrt{\left(x_{i}-x_{o}-z_{i} \cdot \sin \beta_{x}\right)^{2} \cdot \cos ^{2} \beta_{x}+\left(y_{i}-y_{o}-z_{i} \cdot \sin \beta_{y}\right)^{2} \cdot \cos ^{2} \beta_{y}}
$$

where $\boldsymbol{R}$ is mid internal radius of the tank cylindrical part;

$\boldsymbol{x}_{\boldsymbol{o}}, \boldsymbol{y}_{\boldsymbol{o}}$ are horizontal coordinates of the point on the tank axis if $\boldsymbol{z}_{\boldsymbol{o}}=0$;

$\boldsymbol{\beta}_{\boldsymbol{x}}, \boldsymbol{\beta}_{\boldsymbol{y}}$ are the tank axis angles tilt in coordinates plane projection $\boldsymbol{x} \boldsymbol{z}$ and $\boldsymbol{y} \boldsymbol{z}$.

For vertical tank in formulas that calculate it interval capacities (8) and surface areas (10) we use only three geometrical parameters:

$$
\begin{gathered}
V_{z_{f}}=\pi \cdot R^{2} \cdot \sqrt{1+\operatorname{tg}^{2} \beta_{x}+\operatorname{tg}^{2} \beta_{y}} \cdot z_{f}, \\
S_{z_{f}}=\pi \cdot R \cdot\left(1+\sqrt{1+\operatorname{tg}^{2} \beta_{x}+\operatorname{tg}^{2} \beta_{y}}\right) \cdot z_{f}
\end{gathered}
$$

For simplication of perception another important corrections such as corrections for tank deformations due to liquid, correction for tank deformation due to temperature variations and others have not been included.

\section{Conclusions:}

1. On the example of the vertical cylindrical tanks it has been proposed mathematically strict generalized method of evaluation of total and interval tank capacities, geometrical parameters of surfaces were obtained from approximation by LSM.

2. Tank geometrical parameters obtained by approximation results are correlated, that lead to evaluation of interval capacity uncertainty with their correlation matrix obtained by LSM.

3. It has been proposed universal algorithm of correction calculations to interval capacities of any tank surfaces for deviation of real shape surface from approximate. 
4. It has been developed general theoretical method of evaluation interval capacity uncertainty of any tank, that provide adequate comparable results without regard to tanks shape and dimension, their surface deformations, geodetic measurement accuracy, and also points location and quantity, which coordinates were determined on the surfaces. 